\title{
Curative Multicycle Radioimmunotherapy Monitored by Quantitative SPECT/CT-Based Theranostics, Using Bispecific Antibody Pretargeting Strategy in Colorectal Cancer
}

\author{
Sarah M. Cheal ${ }^{1,2}$, Edward K. Fung ${ }^{3}$, Mitesh Patel ${ }^{2}$, Hong Xü $\mathrm{Xu}^{4}$ Hong-fen $\mathrm{Guo}^{4}$, Pat B. Zanzonico ${ }^{3}$, Sebastien Monette ${ }^{5}$, \\ K. Dane Wittrup ${ }^{6-8}$, Nai-Kong V. Cheung ${ }^{2,4}$, and Steven M. Larson ${ }^{1,2}$ \\ ${ }^{I}$ Department of Radiology, Memorial Sloan Kettering Cancer Center, New York, New York; ${ }^{2}$ Molecular Pharmacology Program, \\ Memorial Sloan Kettering Cancer Center, New York, New York; ${ }^{3}$ Department of Medical Physics, Memorial Sloan Kettering Cancer \\ Center, New York, New York; ${ }^{4}$ Department of Pediatrics, Memorial Sloan Kettering Cancer Center, New York, New York; ${ }^{5}$ Tri- \\ Institutional Laboratory of Comparative Pathology, Memorial Sloan Kettering Cancer Center, Weill Cornell Medicine, and The \\ Rockefeller University, New York, New York; ${ }^{6}$ Department of Chemical Engineering, Massachusetts Institute of Technology, \\ Cambridge, Massachusetts; ${ }^{7}$ Department of Biological Engineering, Massachusetts Institute of Technology, Cambridge, \\ Massachusetts; and ${ }^{8}$ Koch Institute for Integrative Cancer Research, Massachusetts Institute of Technology, Cambridge, \\ Massachusetts
}

Radioimmunotherapy of solid tumors using antibody-targeted radionuclides has been limited by low therapeutic indices (TIs). We recently reported a novel 3-step pretargeted radioimmunotherapy (PRIT) strategy based on a glycoprotein A33 (GPA33)-targeting bispecific antibody and a small-molecule radioactive hapten, a complex of ${ }^{177} \mathrm{Lu}$ and S-2-(4-aminobenzyl)-1,4,7,10-tetraazacyclododecane tetraacetic acid ( $\left.{ }^{177} \mathrm{Lu}-\mathrm{DOTA}-\mathrm{Bn}\right)$, that leads to high Tls for radiosensitive tissues such as blood $(T I=73)$ and kidney $(T I=12)$. We tested our hypothesis that a fractionated anti-GPA33 DOTAPRIT regimen calibrated to deliver a radiation absorbed dose to tumor of more than 100 Gy would lead to a high probability of tumor cure while being well tolerated by nude mice bearing subcutaneous GPA33-positive SW1222 xenografts. Methods: We treated groups of nude mice bearing 7-d-old SW1222 xenografts with a fractionated 3-cycle anti-GPA33 DOTA-PRIT regimen (total administered ${ }^{177} \mathrm{Lu}$-DOTA-Bn activity, $167 \mathrm{MBq} /$ mouse; estimated radiation absorbed dose to tumor, $110 \mathrm{~Gy}$ ). In randomly selected mice undergoing treatment, serial SPECT/CT imaging was used to monitor treatment response and calculate radiation absorbed doses to tumor. Necropsy was done on surviving animals 100-200 d after treatment to determine frequency of cure and assess select normal tissues for treatment-related histopathologies. Results: Rapid exponential tumor progression was observed in control treatment groups (i.e., no treatment or ${ }^{177} \mathrm{Lu}$-DOTA-Bn only), leading to euthanasia due to excessive tumor burden, whereas 10 of 10 complete responses were observed for the DOTA-PRIT-treated animals within $30 \mathrm{~d}$. Treatment was well tolerated, and 100\% histologic cure was achieved in 9 of 9 assessable animals without detectable radiation damage to critical organs, including bone marrow and kidney. Radiation absorbed doses to tumor derived from SPECT/CT (102 Gy) and from biodistribution ( $110 \mathrm{~Gy}$ ) agreed to within $6.9 \%$. Of the total dose of approximately $100 \mathrm{~Gy}$, the first dose contributes $30 \%$, the second dose $60 \%$, and the third dose $10 \%$. Conclusion: In a GPA33-positive human colorectal cancer xenograft mouse model, we validated a SPECT/CT-based theranostic PRIT regimen that led

Received Mar. 13, 2017; revision accepted May 31, 2017.

For correspondence or reprints contact: Steven M. Larson, Memorial Sloan Kettering Cancer Center, 415 East 68th St., Z-2064, New York, NY 10065.

E-mail: larsons@mskcc.org

Published online Jul. 13, 2017.

COPYRIGHT @ 2017 by the Society of Nuclear Medicine and Molecular Imaging. to $100 \%$ complete responses and $100 \%$ cures without any treatmentrelated toxicities, based on high Tls for radiosensitive tissues. These studies support the view that anti-GPA33 DOTA-PRIT will be a potent radioimmunotherapy regimen for GPA33-positive colorectal cancer tumors in humans.

Key Words: pretargeting; colorectal cancer; radioimmunotherapy; SPECT

J Nucl Med 2017; 58:1735-1742

DOI: 10.2967/jnumed.117.193250

$\mathbf{R}$ adioimmunotherapy has been investigated for decades as a promising therapeutic strategy for selective targeting of ionizing radioisotopes to liquid and solid tumors in humans (1). Few successes in safely and effectively treating tumors by this approach have been achieved in solid tumors (1). An effective radioimmunotherapy approach would address a major unmet need, especially for patients with advanced metastatic colorectal cancer (CRC), a cancer with a 5 -y survival of only $11 \%$. Unfortunately, numerous clinical investigations of radioimmunotherapy against CRC-associated antigens have yielded limited clinical benefit, irrespective of radioisotope used (1). The main hurdle observed was insufficient radiation delivery to tumor at radioimmunotherapy activities compatible with body tolerance. Maximal treatment benefits reported thus far using radioimmunotherapy with whole $\operatorname{IgG}$ have been stabilized tumor growth in a minority of treated patients, and calculated radiation-absorbed doses have been relatively low; for example, the use of ${ }^{131}$ I-huA33 to target GPA33 in CRC resulted in modest tumor doses ranging from 12 to $33 \mathrm{~Gy}$ at the maximum tolerated dose of $1,480 \mathrm{MBq} / \mathrm{m}^{2}$, without cure in small tumors, and with a corresponding mean red marrow-absorbed dose of approximately 122 cGy (therapeutic index $[\mathrm{TI}]=\sim 16)(2,3)$.

TI in radioimmunotherapy can be greatly improved using a multistep method, such as the pretargeting approach, based on the discovery of Reardan, et al., that antibodies could be developed against metal chelates (4). This allowed for the separation of the antibody-based tumor-targeting step from the radioactive 
small molecular hapten component. Additional improvements in targeting to tumor were achieved by modifications, such as the use of streptavidin by Axworthy et al. (5). However, these ultimately failed based on serious practical drawbacks related to immunogenicity of reagents and low TI (1).

In response to these limitations, Orcutt et al. used mathematic modeling to estimate that a hapten-binding antibody must have an affinity of less than $100 \mathrm{pM}$ for efficient delivery of ionizing radiation in pretargeted radioimmunotherapy (PRIT) (6) and developed a novel PRIT approach called DOTA-PRIT (Fig. 1). The primary features include that the modular bispecific antibody format (IgG-scFv) offers multivalent and simultaneous antibody binding of 2 distinct antigens: a tumor antigen and the pM affinity anti-DOTA hapten antibody fragment $\mathrm{C} 825$ that recognizes small radioactive yttriumor lutetium-chelate complexes of DOTA-Bn (6); and the use of radio-haptens (e.g., ${ }^{177} \mathrm{Lu}-\mathrm{DOTA}-\mathrm{Bn}$ ) with almost exclusive renal clearance and with negligible retention in normal tissue (7)).

The purpose of this study was to investigate the efficacy and toxicity of a 3-cycle fractionated DOTA-PRIT regimen capable of delivering an absorbed dose of more than 100 Gy to subcutaneous human CRC tumor xenografts. We report our results with ${ }^{177} \mathrm{Lu}$, a theranostic isotope that enables noninvasive treatment monitoring and dosimetry in combination with therapeutic $\beta$-emission (half-life $=6.7 \mathrm{~d}$; maximum energy of the primary [78.6\%] $\beta$-emission from ${ }^{177} \mathrm{Lu}=0.5 \mathrm{MeV}$; average energy of the primary $\beta$-emission from ${ }^{177} \mathrm{Lu}=0.13 \mathrm{MeV}$; maximum range $=$ $1.5 \mathrm{~mm}$ ). The $\gamma$-emissions of ${ }^{177} \mathrm{Lu}(208$ and $113 \mathrm{keV}$, with $10 \%$ and $6 \%$ abundance, respectively) enable direct quantitative theranostic SPECT imaging during treatment. A subaim was to validate the SPECT imaging component of DOTA-PRIT for direct tumor dosimetry, on a treatment cycle-specific basis.

\section{MATERIALS AND METHODS}

\section{Reagents and General Procedures}

The anti-GPA33 DOTA-PRIT bispecific antibody huA33-C825 $(210 \mathrm{kDa})$ was expressed and purified as described by Cheal et al. (8). A treatment cycle of anti-GPA33 DOTA-PRIT consisted of 3 separate intravenous injections via the tail vein: $0.25 \mathrm{mg}$ of huA33$\mathrm{C} 825 \mathrm{at}-28 \mathrm{~h}$, then $62.5 \mu \mathrm{g}$ of clearing agent at $-4 \mathrm{~h}$, and $55 \mathrm{MBq}$ of ${ }^{177} \mathrm{Lu}-\mathrm{DOTA}-\mathrm{Bn}$ at $0(8)$. The GPA33-postive human CRC cell line SW1222 was obtained from the Ludwig Institute for Cancer Immunotherapy and grown by serial passage. All animal experiments were done in accordance with protocols approved by the Institutional Animal Care and Use Committee of Memorial Sloan Kettering Cancer Center, following National Institutes of Health guidelines for animal welfare. Technical details regarding cell culture and animal models were previously described by Cheal et al. (8).

\section{DOTA-PRIT Regimen}

Mice bearing subcutaneous SW1222 xenografts $\left(30-170 \mathrm{~mm}^{3}, 7 \mathrm{~d}\right.$ after inoculation) with either no treatment $(n=5$; tumor volume $=$ $\left.114 \pm 35 \mathrm{~mm}^{3}\right),{ }^{177} \mathrm{Lu}$-DOTA-Bn only $(n=5$; tumor volume $=128 \pm$ $34 \mathrm{~mm}^{3} ; 2$ cycles, total administered ${ }^{177}$ Lu-DOTA-Bn activity: 110 $\mathrm{MBq}$ ), or a 3-cycle DOTA-PRIT regimen consisting of anti-GPA33 PRIT + 55.5 MBq of ${ }^{177} \mathrm{Lu}-\mathrm{DOTA}-\mathrm{Bn}(n=10$; tumor volume $=83 \pm$ $38 \mathrm{~mm}^{3}$; total administered ${ }^{177} \mathrm{Lu}-\mathrm{DOTA}-\mathrm{Bn}$ activity/mouse: $167 \mathrm{MBq}$ ) were studied. For DOTA-PRIT, cycle 1 was given on days 7 and 8 after tumor inoculation. This was repeated $6 \mathrm{~d}$ later (i.e., weekly), with cycle 2 given on days 14 and 15 and cycle 3 given on days 21 and 22 . All groups were monitored 2-3 times per week for overall health, body weight, and measurement of tumor burden by external caliper, with tumor volume calculated using the volume of an ellipsoid. Mice were sacrificed when the tumor volume exceeded $2,500 \mathrm{~mm}^{3}$ (or earlier if the tumor burden interfered with mobility), or if excessive weight loss $(>25 \%)$ from pretreatment baseline was noted. Tumor growth was analyzed by performing a nonlinear regression fit of an exponential growth curve to the tumor volume data collected in the first $21 \mathrm{~d}$ after tumor inoculation for the nontreated and treatment with ${ }^{177} \mathrm{Lu}$-DOTA-Bnonly groups. The tumor growth data of the animals treated with the 3-cycle DOTA-PRIT regimen were fitted by an initial exponential growth curve up to and including day 14 (i.e., onset time of growth delay and shrinkage) using GraphPad Prism (version 6.00).

\section{Monitoring of DOTA-PRIT with SPECT/CT}

All SPECT/CT scans were obtained using a dedicated small-animal scanner (NanoSPECT/CT; Bioscan) with an animal palette heated to $37^{\circ} \mathrm{C}$. Animals were anesthetized during scanning using a mixture of $1.5 \%-2 \%$ isoflurane (Baxter Healthcare) and oxygen gas. A CT topogram was acquired first, followed by a $360^{\circ}$ small-animal SPECT using a 4-head $\gamma$-camera with pinhole collimators $(1.4 \mathrm{~mm})$. The SPECT scan time was adjusted for each mouse to record approximately 30,000 counts minimum per frame (20-55 min). Bioscan HiSPECT software was used for iterative image reconstruction and fusion of CT and SPECT images.

Five of ten of the animals undergoing DOTA-PRIT were randomly selected for serial noninvasive SPECT/CT imaging for verification of tumor targeting and calculation of tumor dosimetry. Because of the length of time required for each scan (30-40 min per mouse), the imaging field of view was limited to the caudal half of the animal (midline to tail). Each animal was imaged 5-6 times at various time points during fractionated treatment, up to $24 \mathrm{~h}$ after injection of cycle 3 with ${ }^{177}$ Lu-DOTA-Bn (day 23) (Fig. 2). For each image, tumor volumes were estimated by CT image analysis, and the total activity in the tumor region was estimated by SPECT image analysis. Tumor count rates were converted to activity concentrations $\left(\mathrm{MBq}\right.$ per $\mathrm{mm}^{3}$ or gram $[\mathrm{g}]$ ) using the measured system calibration factor for ${ }^{177} \mathrm{Lu}$.

To determine whether partial-volume correction would be necessary, a phantom consisting of 4 fillable spheres with diameters of 5.8, 7.8, 9.9, and $12.4 \mathrm{~mm}$ was imaged on the SPECT/CT with a solution of ${ }^{177} \mathrm{Lu}$ having a nominal concentration of $3.7 \mathrm{MBq}(0.1 \mathrm{mCi}) / \mathrm{mL}$. Regions of interest were drawn on the coregistered CT images and activity within the spheres measured in the reconstructed SPECT images. A diameter-dependent recovery curve was fitted and compared with the CT-measured tumor sizes. A partial-volume effect was not observed in the phantom SPECT images above a size of approximately $200 \mathrm{~mm}^{3}$. For the measured tumor sizes, we estimated a minimum recovery coefficient of 0.86 for cycle 1 and 0.71 for cycle 3 . Because most tumor sizes were greater than about $200 \mathrm{~mm}^{3}$ for the duration of the experiment, we did not apply a partial-volume correction to the results.

\section{SPECT Dosimetry}

${ }^{177} \mathrm{Lu}$-DOTA-Bn activity concentration was measured by SPECT/CT imaging of the SW1222 xenograft mice. Activity concentrations as a function of time without decay correction for cycles 1 and 2 were fit to a single exponential. The apparent half-life for this exponential therefore incorporated both physical and biologic decay effects.

Durational cumulated activity was calculated for each cycle from 0 to $160 \mathrm{~h}$ after the start of cycle. Durational cumulated activity for cycle 2 therefore includes residual activity from cycle 1 . Activity for cycle 3 was extrapolated by assuming an exponential with the same effective half-life as cycle 2 . This assumption is based on the fact that cycle 3 should behave similarly to cycle 2 as both start while there is residual activity still present from the previous treatment cycle. Total 


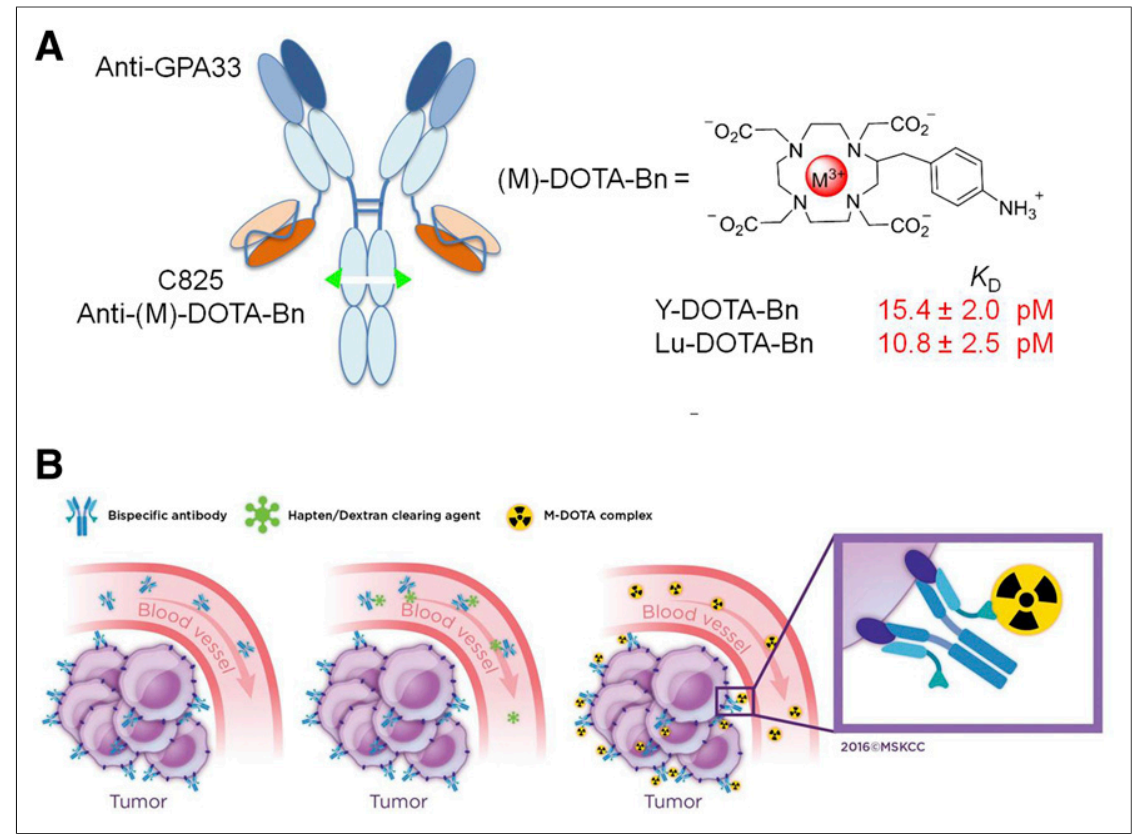

FIGURE 1. Anti-GPA33 DOTA-PRIT concept. (A) Schematic representation of anti-GPA33 BsAb huA33-C825 (left) and M-DOTA-Bn hapten (right), vehicle by which ${ }^{177} \mathrm{Lu}$ treatment is administered. Anti-(M)-DOTA-Bn scFv antibody $\mathrm{C} 825$ has approximately equal low-pM affinity for either Y-DOTA-Bn or Lu-DOTA-Bn. (B) Schematic representation of 3-step anti-GPA33 DOTA-PRIT protocol. (Copyright 2016, reprinted with permission from Memorial Sloan Kettering Cancer Center $[\mathrm{MSK}]$.)

cumulated activities were also calculated for each cycle from the start of the multicycle protocol, assuming no further treatment and complete elimination of the tumor at $496 \mathrm{~h}$ after injection of ${ }^{177} \mathrm{Lu}$ activity in cycle 1 . Total cumulated activity for cycle 1 represents dose delivered from 1 injection from 0 to 496 hours after injection. Total cumulated activity for cycle 2 represents dose delivered from the first and second injections (but not the third) assuming the same rate of decay for cycle 2 to $496 \mathrm{~h}$.

Tumor time-activity curves were also simulated for the 3-cycle treatment regimen. Curve parameters from the exponential fit to the first cycle of each subject were used to generate the corresponding second and third cycle curves. Residual activity from each previous cycle was taken into account in the initial activity at the beginning of each cycle. Elimination of radiolabel was assumed to proceed at the same exponential rate as for the first cycle. Mean tumor-absorbed doses were calculated as the product of the cumulated activities thus determined and the average energy emitted per decay of ${ }^{177} \mathrm{Lu}$ in the form of $\beta$-particles, assuming complete local absorption of the $\beta$-particles and ignoring the dose contribution of the much more penetrating $\gamma$-rays.

\section{Necropsy of Animals That Underwent DOTA-PRIT}

Mice were euthanized with $\mathrm{CO}_{2}$ according to the guidelines of the American Veterinary Medical Association. After gross examination, organs were fixed in $10 \%$ neutral buffered formalin. After fixation, bones were decalcified in a formic acid solution (Surgipath Decalcifier I; Leica Biosystems). Fixed organs were further processed for histology in ethanol and xylene in a Leica ASP6025 tissue processor and embedded in paraffin. Paraffin blocks were sectioned at $5 \mu \mathrm{m}$, stained with hematoxylin and eosin, and examined by a board-certified veterinary pathologist. The following tissues were examined: skin and subcutaneous tissue from tumor implantation site, liver, kidney, spleen, and bone marrow (sternum, vertebrae, femur, and tibia). Any abnormal gross or histologic findings were noted.

\section{Hematology and Serum Chemistry}

For hematology, blood was collected into tubes containing EDTA. Automated analysis was performed by the LCP on an IDEXX Procyte DX hematology analyzer, and the following parameters were determined: white blood cell count, red blood cell count, hemoglobin concentration, hematocrit, mean corpuscular volume, mean corpuscular hemoglobin, mean corpuscular hemoglobin concentration, red blood cell distribution width standard deviation and coefficient of variance, platelet count, platelet distribution width, mean platelet volume, and relative and absolute counts of neutrophils, lymphocytes, monocytes, eosinophils, basophils, and reticulocytes.

For serum chemistry, blood was collected into tubes containing a serum separator and then centrifuged to obtain serum for analysis. Serum chemistry was performed by the LCP on a Beckman Coulter AU680 analyzer for the following parameters: alkaline phosphatase concentration, alanine aminotransferase concentration, aspartate aminotransferase concentration, creatine kinase concentration, gamma-glutamyl transpeptidase concentration, albumin concentration, total protein concentration, globulin concentration, albumin/globulin ratio, total bilirubin concentration, blood urea nitrogen concentration, creatinine concentration, cholesterol concentration, triglycerides concentration, glucose concentration, calcium concentration, phosphorus concentration, chloride concentration, potassium concentration $(\mathrm{K})$, sodium concentration $(\mathrm{Na}), \mathrm{Na} / \mathrm{K}$ ratio.

\section{Statistical Analysis}

Kaplan-Meier survival analysis or statistical comparisons between groups (Student $t$ test) were performed with Prism software (version 6.0; GraphPad Software). For all studies, the level of statistical significance is set at a $P$ level of less than 0.05 .

\section{RESULTS}

\section{Fractionated Treatment with DOTA-PRIT}

No significant difference in average tumor volumes was found among the 3 groups at the start of treatment. Tumor sizes measured

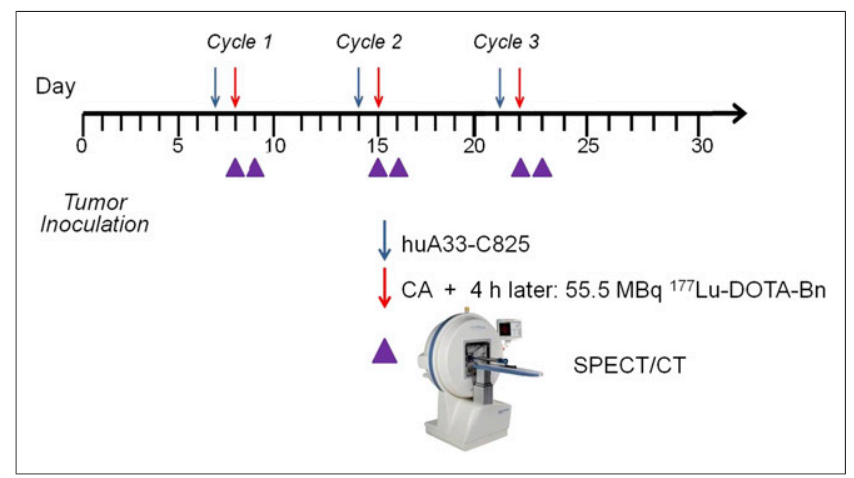

FIGURE 2. Timeline of theranostic anti-GPA33 DOTA-PRIT. 
over the course of the experiment demonstrated a clear difference in efficacy between the control treatment groups (i.e., no treatment or ${ }^{177} \mathrm{Lu}-\mathrm{DOTA}-\mathrm{Bn}$ only) and the DOTA-PRIT-treated groups. This is shown in Figure 3A where tumor volumes are plotted as a function of time after xenograft inoculation for all treatment groups.

Kaplan-Meier plots (Fig. 3B) were generated for all 3 groups. Development of a $1,000 \mathrm{~mm}^{3}$ tumor volume that necessitated euthanasia due to excessive tumor burden was designated as the survival endpoint. All untreated mice and mice treated with ${ }^{177} \mathrm{Lu}-$ DOTA-Bn only $(10 / 10,100 \%)$ developed large, exponentially growing tumors, requiring euthanasia of the animals. Individual tumor doubling times ranged from 3.6 to $6.0 \mathrm{~d}\left(R^{2}=0.90-0.99\right)$. Because of this rapid tumor growth, treatment with ${ }^{177} \mathrm{Lu}$-DOTA-Bn only was limited to just 2 cycles. The median time to a terminal $1,000 \mathrm{~mm}^{3}$ tumor volume was $19 \mathrm{~d}$ for no treatment and $16 \mathrm{~d}$ for treatment with 2 cycles of ${ }^{177} \mathrm{Lu}-\mathrm{DOTA}-\mathrm{Bn}$ only. For all DOTAPRIT-treated mice $(10 / 10,100 \%)$, the tumors grew exponentially to a maximum size of $247-386 \mathrm{~mm}^{3}$ on day 14 (tumor doubling time range, $\left.2.2-5.1 \mathrm{~d} ; R^{2}=0.82-0.99\right)$. After the third cycle of treatment (administered on days 21 and 22, after tumor inoculation), tumors showed rapid regression to below the palpable threshold $\left(\leq 10 \mathrm{~mm}^{3}\right)$ by approximately day 30 (Fig. 3A). Tumor volumes at the start of cycles 2 and 3 were $298 \pm 51 \mathrm{~mm}^{3}$ and $31 \pm 19 \mathrm{~mm}^{3}$, respectively, measured manually by caliper. For the DOTA-PRIT-treated mice, median time to the terminal $1,000 \mathrm{~mm}^{3}$ tumor burden was never reached because all animals (10/10) showed complete responses with no recurrence before study endpoints (118-200 d after inoculation). By log-rank (Mantel-Cox) test, survival of the DOTA-PRIT group was significant $(P<0.0001)$.

\section{SPECT/CT Imaging to Monitor Treatment and Quantify Tumoricidal Tumor Doses}

SPECT/CT of tumor-bearing mice undergoing treatment demonstrated highly selective tumor localization of pretargeted ${ }^{177} \mathrm{Lu}-$ DOTA-Bn as early as $1 \mathrm{~h}$ after injection of ${ }^{177} \mathrm{Lu}-\mathrm{DOTA}-\mathrm{Bn}$. At this time, the average effective ${ }^{177} \mathrm{Lu}$ activity concentration in tumors (as average $\mathrm{MBq} / \mathrm{g} \pm \mathrm{SD} ; n=4$ ) was $3.92 \pm 0.85 \mathrm{MBq} / \mathrm{g}$, corresponding to an uptake of injected ${ }^{177} \mathrm{Lu}$-DOTA-Bn of $7.1 \% \pm 1.5 \%$ per $g$ of tumor volume. Prolonged and high tumor retention of ${ }^{177} \mathrm{Lu}$ activity was also observed at 24 and $160 \mathrm{~h}$ after injection (non-decaycorrected activities, $3.17 \pm 1.10$ and $0.64 \pm 0.19 \mathrm{MBq} / \mathrm{g}$ ), suggesting minimal biologic clearance of ${ }^{177} \mathrm{Lu}$ activity from the tumor. Activity in the bladder region is apparent in the SPECT images at $1 \mathrm{~h}$ after injection, consistent with renal clearance of free ${ }^{177} \mathrm{Lu}-\mathrm{DOTA}-\mathrm{Bn}$ (data not shown). Rapid renal clearance of free ${ }^{177} \mathrm{Lu}-\mathrm{DOTA}-\mathrm{Bn}$ was also supported by the observation that whole-body activity of each individual animal, determined by assay in a dose calibrator, was similar to the measured tumor-associated activity. ${ }^{177} \mathrm{Lu}$ activity remaining in the mice measured after 24 and $160 \mathrm{~h}$ after injection of cycle 1 (as average $\pm \mathrm{SD} ; n=5$ ) was $6.98 \% \pm 1.64 \%$ and $1.18 \% \pm 0.39 \%$ of administered activity, respectively. Although the SPECT/CT imaging demonstrates that the most prominent focus of activity was in the tumor, low levels of ${ }^{177} \mathrm{Lu}$ activity were detected in the liver, spleen, and kidney $(\sim 10$ - to 15-fold-less activity than tumor, as percentage injected dose per gram of tissue, based on previous biodistribution studies). For cycle 2 , the whole-body percentage of administered ${ }^{177} \mathrm{Lu}$ activity remaining after 24 and $160 \mathrm{~h}$ after injection was $12.83 \% \pm 2.01 \%$ and $1.98 \% \pm 0.44 \%$, respectively. Finally, for cycle 3 , the

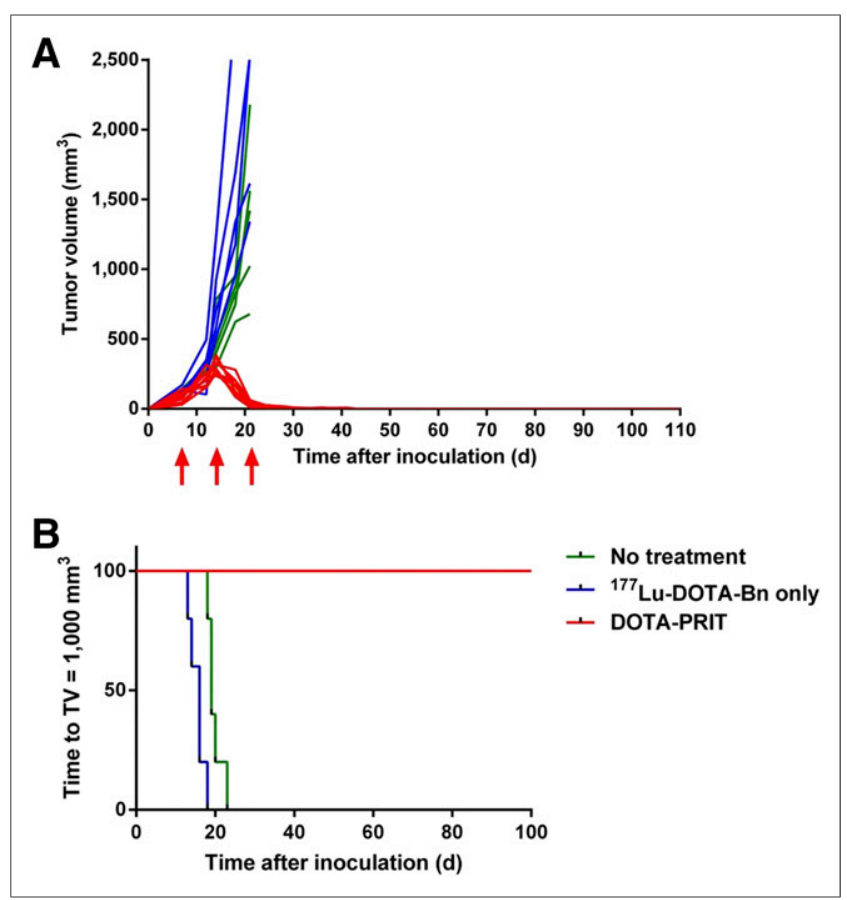

FIGURE 3. Efficacy of DOTA-PRIT evaluated as tumor growth and survival in mice bearing SW1222 subcutaneous xenografts. (A) Tumor growth presented as change in tumor volume for each individual mouse over time (green, no treatment; blue, ${ }^{177} \mathrm{Lu}-\mathrm{DOTA}-\mathrm{Bn}$ only; red, DOTA-PRIT; red arrows, 3-cycle treatment times). (B) KaplanMeier plot of DOTA-PRIT (shown as time to tumor burden of $1,000 \mathrm{~mm}^{3}$ ).

whole-body percentage of administered ${ }^{177} \mathrm{Lu}$ activity remaining after $24 \mathrm{~h}$ was $4.81 \% \pm 1.03 \%$ (no additional data were collected for cycle 3 after $24 \mathrm{~h}$ ).

The CT image-derived tumor volumes (in $\mathrm{mm}^{3}$ ) demonstrating tumor doubling followed by regression are shown in Table 1. SPECT image-derived ${ }^{177} \mathrm{Lu}$ activity estimates for tumor regions of interest are shown in Table 2 and Figure 4. Tumor dosimetry was calculated from ${ }^{177} \mathrm{Lu}$ time-activity curves and is presented in Table 3. Representative serial SPECT/CT images originating from the same animal are provided in Figure 5. The contribution to total dose was estimated to be greatest during cycle 2 (average dose during cycle 2 period, $43.8 \mathrm{~Gy} ; n=5$ ). The fitted exponential decay curves of the 3-cycle treatment regimen are shown in Figure 6 , illustrating the difference between the measured activity and expected activity from single and 2-cycle protocols. Total dose from the 3-cycle treatment was estimated at 102 Gy. Radiation absorbed doses to tumors derived from SPECT/CT (102 Gy) and biodistribution (109.6 Gy) agreed to within $6.9 \%$. For comparison, ${ }^{177} \mathrm{Lu}$ activity in the tumor for a 3-cycle treatment was simulated on the basis of kinetics derived only from the first cycle of the experimental data. The time-activity curves are shown in Supplemental Figure 1 (supplemental materials are available at http://jnm.snmjournals.org), with tumor dose estimates calculated from the curves shown in Supplemental Table 1. Total tumor dose predicted by the simulation was about $92 \mathrm{~Gy}$, assuming first-cycle kinetics throughout, that is, the kinetics of subsequent cycles essentially ignore the effects of previous cycles. Measured dose from the 3-cycle treatment was higher than predicted. 
TABLE 1

CT Image-Derived Tumor Volumes $\left(\mathrm{mm}^{3}\right)$

\begin{tabular}{|c|c|c|c|c|c|c|}
\hline \multirow[b]{2}{*}{ Mouse } & \multicolumn{3}{|c|}{ Cycle 1} & \multicolumn{2}{|c|}{ Cycle 2} & \multirow[b]{2}{*}{$\begin{array}{l}\text { Cycle } 3,24 \mathrm{~h} \\
\text { after injection }\end{array}$} \\
\hline & $\begin{array}{l}1 \mathrm{~h} \text { after } \\
\text { injection }\end{array}$ & $\begin{array}{l}24 \mathrm{~h} \text { after } \\
\text { injection }\end{array}$ & $\begin{array}{l}160 \mathrm{~h} \text { after } \\
\text { injection }\end{array}$ & $\begin{array}{l}24 \mathrm{~h} \text { after } \\
\text { injection }\end{array}$ & $\begin{array}{l}60 \mathrm{~h} \text { after } \\
\text { injection }\end{array}$ & \\
\hline 1 & 219 & 238 & 486 & 398 & 120 & 97.9 \\
\hline 2 & 168 & 267 & 373 & 318 & 166 & 137 \\
\hline 3 & 140 & 212 & 206 & 192 & 94.3 & 76.8 \\
\hline 4 & 147 & 212 & 193 & 186 & 113 & 99.6 \\
\hline 5 & NA & 297 & 291 & 293 & 128 & 94 \\
\hline Average & 169 & 245 & 310 & 278 & 124 & 101 \\
\hline SD & 35.7 & 36.8 & 122 & 89.6 & 26.5 & 22.0 \\
\hline
\end{tabular}

\section{Toxicity of Fractionated DOTA-PRIT}

All treatments were well tolerated, with no average weight loss greater than $10 \%$ from baseline observation (Supplemental Fig. 2), suggesting no acute toxicity. For DOTA-PRIT-treated mice, a slight drop in average weight $(\sim 5 \%)$ from baseline was observed for the duration of treatment administration, but was reversible to pretreatment weight about $10 \mathrm{~d}$ after the final ${ }^{177} \mathrm{Lu}-\mathrm{DOTA}-\mathrm{Bn}$ injection. Weight loss was most likely the result of a transient and reversible gastrointestinal toxicity, which did not leave permanent histologic changes, but formal toxicity studies are warranted to verify this. No early, treatment-related deaths were observed.

Histopathology confirmed complete tumor disappearance in all surviving DOTA-PRIT-treated mice (9/9) at 3-6 mo (96-178 d) after treatment. No evidence of tumors was observed macroscopically or histologically at the site of tumor inoculation or in distant organs in 9 of 9 mice that underwent necropsy, consistent with histologic complete cures in those animals $(100 \%)$.

We found no evidence of treatment-related radiotoxicity in radiosensitive organs in treated animals at either 3 or 6 mo after treatment. For example, in the 5 mice submitted for necropsy and histopathology assessment at 3 mo after treatment, no abnormalities, such as bone marrow depletion as seen in ${ }^{177} \mathrm{Lu}-\mathrm{IgG}$ radioimmunotherapy (9), nephrotoxicity, or increased incidence of mesangial glomerulopathy as seen with ${ }^{177} \mathrm{Lu}$-peptide receptor radiotherapy (10), were observed.

A variety of histopathologic changes were noted; these are presented in Supplemental Table 3. Although most of these findings were compatible with naturally occurring background lesions typical in mice of this strain and age, 2 lesions observed with a low incidence were not typical spontaneous lesions. These 2 lesions, for which a link to test article-induced toxicity could not be ruled out, were mild to moderate granulomatous hepatitis or splenitis (2/9 mice) and marked pulmonary interstitial fibrosis (1/9 mice). Sudden deterioration or death was observed in 2 of 10 treated animals 136 and $158 \mathrm{~d}$ after treatment. In 1 animal, this was determined to be due to pulmonary fibrosis of undetermined cause (as mentioned previously) with secondary myocardial hypertrophy; the other mouse could not be analyzed because of the condition of the animal when it was discovered.

\section{DISCUSSION}

We herein report a 3-cycle, curative theranostic regimen with minimal toxicity for SW1222, a human solid CRC xenograft, derived from a patient with a moderately well-differentiated

TABLE 2

SPECT Image-Derived ${ }^{177}$ Lu Activity Concentrations (Not Decay-Corrected; as MBq/g)

\begin{tabular}{|c|c|c|c|c|c|c|c|c|c|c|c|c|}
\hline Mouse & $\begin{array}{l}\text { Cycle } 1, \\
1 \mathrm{~h} \text { after } \\
\text { injection }\end{array}$ & SD & $\begin{array}{l}\text { Cycle 1, } \\
24 \mathrm{~h} \text { after } \\
\text { injection }\end{array}$ & SD & $\begin{array}{l}\text { Cycle } 1, \\
160 \mathrm{~h} \text { after } \\
\text { injection }\end{array}$ & SD & $\begin{array}{l}\text { Cycle 2, } \\
24 \mathrm{~h} \text { after } \\
\text { injection }\end{array}$ & SD & $\begin{array}{l}\text { Cycle 2, } \\
160 \mathrm{~h} \text { after } \\
\text { injection }\end{array}$ & SD & $\begin{array}{c}\text { Cycle } 3, \\
24 \text { h after } \\
\text { injection }\end{array}$ & SD \\
\hline 1 & 5.14 & 3.30 & 3.81 & 1.77 & 0.59 & 0.41 & 4.00 & 1.64 & 2.23 & 1.60 & 2.54 & 1.84 \\
\hline 2 & 3.96 & 2.25 & 2.50 & 1.42 & 0.55 & 0.38 & 5.22 & 2.41 & 2.71 & 2.52 & 3.55 & 2.38 \\
\hline 3 & 3.37 & 2.20 & 2.72 & 1.72 & 0.96 & 0.63 & 3.26 & 2.08 & 1.69 & 1.41 & 2.39 & 1.69 \\
\hline 4 & 3.24 & 1.94 & 2.05 & 1.50 & 0.62 & 0.42 & 4.00 & 2.53 & 1.73 & 1.96 & 2.60 & 2.48 \\
\hline 5 & NA & NA & 4.74 & 1.38 & 0.48 & 0.37 & 4.22 & 2.82 & 2.28 & 2.36 & 3.05 & 2.42 \\
\hline
\end{tabular}

$\mathrm{NA}=$ data not collected. 


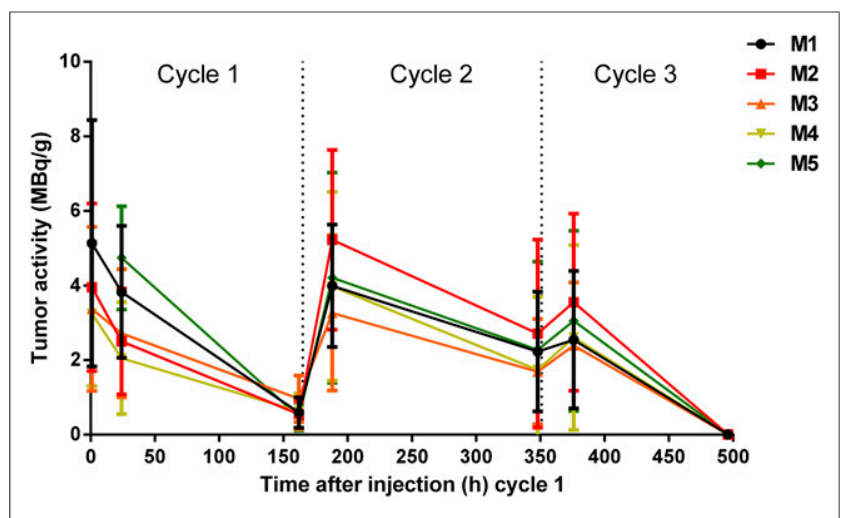

FIGURE 4. Effective (non-decay-corrected) ${ }^{177}$ Lu activity concentrations (as $\mathrm{MBq} / \mathrm{g}$ ) determined using noninvasive theranostic SPECT/CT imaging during treatment.

adenocarcinoma of the colon (classification, Dukes' stage C, a locally invasive cancer). There have been extensive preclinical studies with multistep regimens, but the outcome we report, of a high probability (100\%) of histologic cures without detectable radiosensitive organ toxicity, is to the best of our knowledge, extremely unusual. Our preclinical development focus has been on hitting therapeutic benchmarks, which must be achieved in human translational trials as well. These benchmarks include, for example, cumulative doses to solid tumor of more than $100 \mathrm{~Gy}$ and TIs for radiosensitive tissues of more than 12 for the kidneys, 70 for bone marrow, and 40 for colon. We chose these benchmarks based on clinical human normal-tissue radiation dose tolerance estimates (11), as well as experience gleaned from radiopeptide treatments and related toxicities in humans (1).

In our preliminary studies with anti-GPA33 DOTA-PRIT and SW1222, dose escalation resulted in progressive tumor response, and that toxicity was not limiting (8). At lower tumor doses $(\sim 40-70 \mathrm{~Gy})$, treatment led to complete responses (e.g., we observed $4 / 4$ complete responses for a 2-cycle treatment regimen of $55.5 \mathrm{MBq}$ per cycle) but was ultimately subcurative and resulted in 2 distinct recurrence phenotypes: outgrowth of antigen-positive tumor with the same rate of growth as controls (seen in 3/4); and a histologic senescence pattern that resulted in dormant tumor, in which antigenexpressing cell growth arrested after 1-2 doublings (seen in 1/4; observed at $140 \mathrm{~d}$ after injection). It is critical to assess long-term outcome after radioimmunotherapy, because we and others (12) have shown that late recurrence can occur in this model, with relatively high frequency when treatment is suboptimal. In this study, we found that repeated dose cycles to higher tumor doses were required to achieve $100 \%$ histologic cures in 9 of 9 treated animals available for assessment by necropsy. With the 3-dose regimen, we achieved our targets and in this CRC model, we obtained cures with minimal toxicity (mean TIs for tumor-to-normal tissue were $\sim 73$ [blood], $\sim 10$ [liver], $\sim 10$ [spleen], $\sim 12$ [kidney], and $\sim 110$ [marrowbearing bone (femur)] when 10,000 cGy were delivered to tumor; Tables 1-3 provide dose estimates).

DOTA-PRIT should provide an ideal theranostic platform that permits the use of a single radiopharmaceutical entity for both quantitative imaging and therapy, by simply changing the amount of radioactivity administered. The use of noninvasive SPECT has the advantage of providing a tumor radiation estimate for all 3 doses in the regimen, which is otherwise impossible to achieve by biodistribution (i.e., terminal) studies alone. The absorbed doses to tumor, $10,160 \pm 141 \mathrm{cGy}$, were estimated using SPECT. We defined and derived the dose contribution from each cycle by calculating the total predicted dose after that cycle and subtracting what would have been the total dose, had treatment stopped after the previous cycle. When these contributions as

TABLE 3

Mean Radiation Absorbed Doses to Xenografts from 3-Cycle Pretargeted ${ }^{177}$ Lu-DOTA-Bn Based on Serial SPECT Imaging

\begin{tabular}{|c|c|c|c|c|c|c|}
\hline \multirow[b]{2}{*}{ Mouse } & \multicolumn{2}{|c|}{ Cycle 1} & \multicolumn{2}{|c|}{ Cycle 2} & \multicolumn{2}{|c|}{ Cycle 3} \\
\hline & $\begin{array}{c}\text { Dose } \\
0-160 \text { h (Gy) }\end{array}$ & $\begin{array}{l}\text { Cumulative } \\
\text { dose (Gy) }\end{array}$ & $\begin{array}{c}\text { Dose } \\
\text { 160-320 h (Gy) }\end{array}$ & $\begin{array}{l}\text { Cumulative } \\
\text { dose (Gy) }\end{array}$ & $\begin{array}{c}\text { Dose } \\
320-480 \text { h (Gy) }\end{array}$ & $\begin{array}{l}\text { Cumulative } \\
\text { dose (Gy) }\end{array}$ \\
\hline 1 & 29.1 & 32.8 & 43.5 & 96.0 & 27.7 & 102 \\
\hline 2 & 21.7 & 24.7 & 55.5 & 104 & 37.7 & 117 \\
\hline 3 & 25.9 & 35.4 & 34.6 & 77.4 & 25.4 & 87.5 \\
\hline 4 & 19.9 & 24.4 & 39.9 & 75.7 & 26.1 & 87.4 \\
\hline 5 & 33.4 & 35.8 & 45.3 & 102 & 32.8 & 114 \\
\hline Mean & 26.0 & 30.6 & 43.8 & 91.0 & 29.9 & 102 \\
\hline SD & 5.5 & 5.7 & 7.7 & 13.5 & 5.2 & 14.1 \\
\hline Coefficient of variation & $21 \%$ & $18 \%$ & $18 \%$ & $15 \%$ & $17 \%$ & $14 \%$ \\
\hline
\end{tabular}

Tumors were estimated to be below palpation threshold at $496 \mathrm{~h}$ after injection of cycle 1 , thus having an effective activity concentration of zero. Cumulated dose for each cycle was calculated as total dose after $496 \mathrm{~h}$ after injection of cycle 1 , assuming treatment was stopped at that dose. Therefore, cycle 2 cumulated dose represents expected dose after $496 \mathrm{~h}$ from a treatment comprising only 2 cycles. Dose during the third period was calculated from 320 to $480 \mathrm{~h}$ to be comparable in duration to the other 2 periods. In fact, the period after injection of cycle 3 until complete regression of tumor is slightly longer than the other 2 cycle periods. 


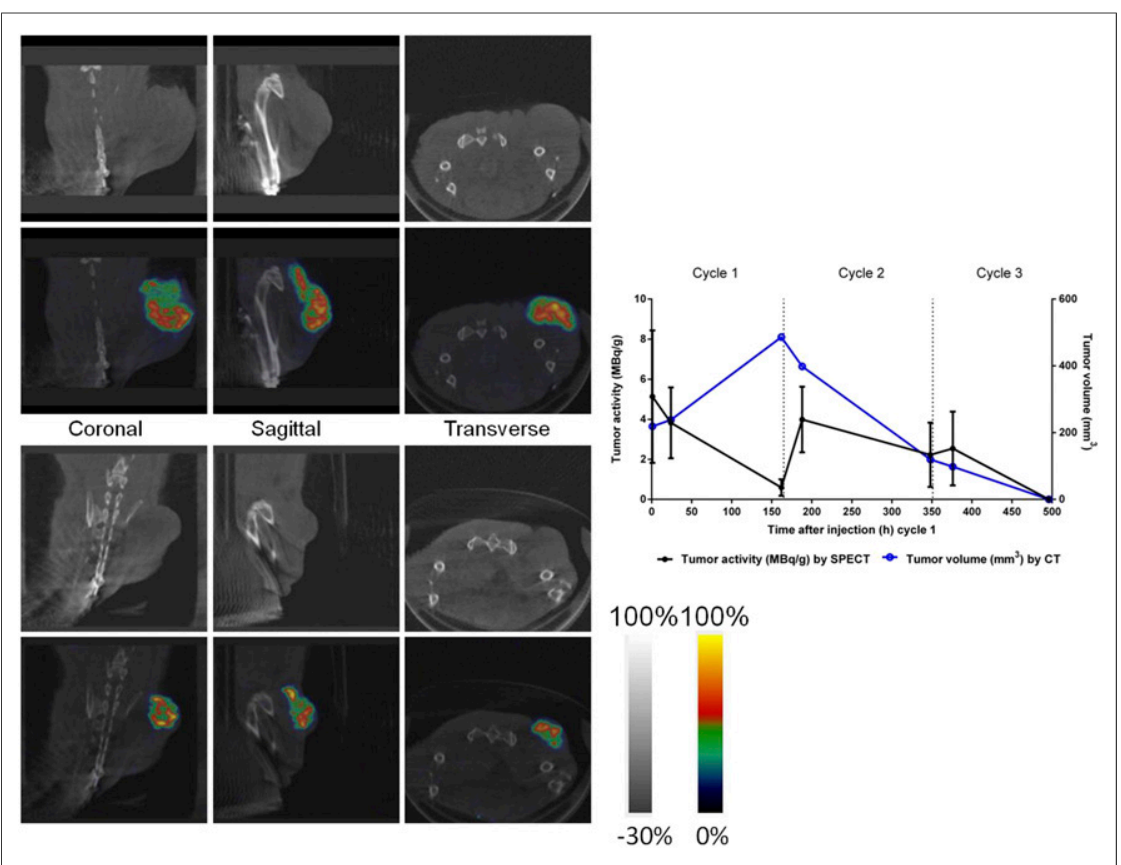

FIGURE 5. (Left) Representative SPECT/CT images obtained from same mouse (M1) at $24 \mathrm{~h}$ after injection of cycle 1 (top left; CT images) and $24 \mathrm{~h}$ after injection of cycle 3 (bottom left; SPECT/CT fusion images). (Right) A graph depicting the effective tumor activity (as $\mathrm{MBq} / \mathrm{g}$ ) derived from SPECT image analysis (right axis) and tumor volume (as $\mathrm{mm}^{3}$ ) derived from CT image analysis (left axis) for this particular animal is provided for reference.

proportions of the total cumulated dose after 3 treatment cycles until total regression of the tumor at $496 \mathrm{~h}$ are considered, it turns out that $60 \%$ of the dose is from the second cycle, and cycles $1(30 \%)$ and $3(10 \%)$ contribute smaller fractions. This result is nonintuitive but is based on dynamic measured changes in tumor volume expansion, which in turn is based on CT during cycle-1 treatment, followed by rapid regression during cycle 2 in particular (Table 1 ).

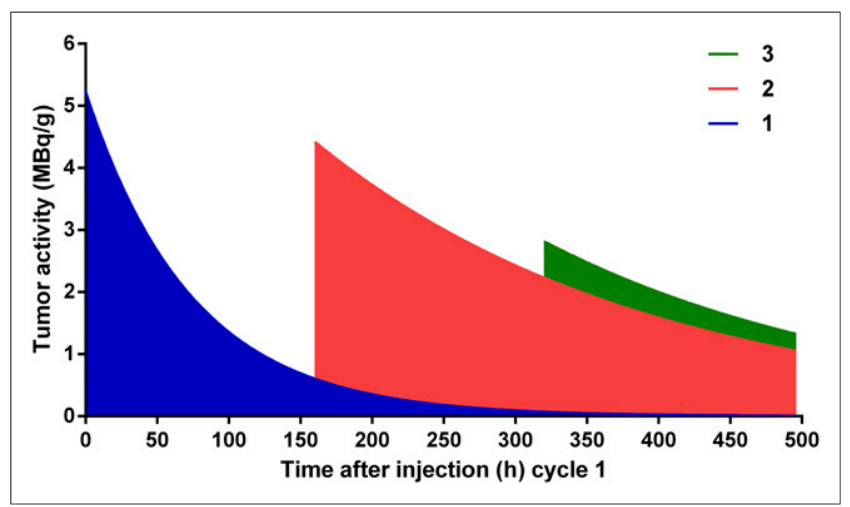

FIGURE 6. Representation of fitted exponential decay curves on 3-cycle treatment regimen. Curves were calculated out to complete removal of radioactive label and truncated at $496 \mathrm{~h}$ after injection of cycle 1 , when tumors were no longer grossly palpable. Cumulated dose for cycle 1 represents dose of blue region, and cumulated dose for cycle 2 includes both blue and red regions. Of total dose of $\sim 100$ Gy, first dose contributes $30 \%$, second dose $60 \%$, and third dose $10 \%$ (Table 2).
As a novel feature of this work, we determined that the addition of cycle 3 contributed $10 \%$ of the total absorbed dose by tumor, which was essential for histologic cure of the 9 of 9 tumors examined after treatment. This result may relate to the state of the treated tumor at the time the third cycle is added. Such information is crucial for later treatment planning in the clinic and underscores the importance of a multiple-cycle treatment regimen that is closely monitored with theranostics.

\section{CONCLUSION}

This study validated a theranostic curative radionuclide therapy regimen with no clear evidence of radiation-induced toxicity in a murine model bearing human CRC solid-tumor xenografts. We demonstrated that 3 cycles were required to achieve tumor doses of more than $100 \mathrm{~Gy}$, and with TIs of greater than 70 for blood and greater than 10 for kidney we observed no radiation-related toxicity despite high total administered ${ }^{177} \mathrm{Lu}$ activity (167 MBq/mouse). Cycle-specific tumor dosimetry was demonstrated using serial SPECT/CT of animals undergoing treatment. Collectively, these preclinical studies support clinical translation of anti-GPA33 DOTA-PRIT.

\section{DISCLOSURE}

This study was supported in part by the Donna \& Benjamin M. Rosen Chair (to Steven M. Larson), Enid A. Haupt Chair (to Nai-Kong V. Cheung), The Center for Targeted Radioimmunotherapy and Theranostics, Ludwig Center for Cancer Immunotherapy of Memorial Sloan Kettering Cancer Center (to Steven M. Larson), and R01-CA101830 (K. Dane Wittrup). Steven M. Larson was also supported in part by P50-CA86438. We also acknowledge the NIH/NCI Cancer Center Support Grant (P30 CA008748) for use of the Tri-Institutional Laboratory of Comparative Pathology, Memorial Sloan Kettering Cancer Center, Weill Cornell Medicine, and The Rockefeller University, New York, NY. Technical services provided by the Memorial Sloan Kettering Small-Animal Imaging Core Facility were also supported by Cancer Center Support Grant P30 CA08748 (to C. Thompson). A Shared Resources Grant from the Memorial Sloan Kettering Cancer Center Metastasis Research Center (to Pat B. Zanzonico), which provided funding for the purchase of the NanoSPECT/CT Plus, is gratefully acknowledged. Steven M. Larson, Nai-Kong V. Cheung, and K. Dane Wittrup own stock in Voreyda Theranostics, Inc. Additionally, Sarah M. Cheal, Hong Xu, Steven M. Larson, and Nai-Kong V. Cheung are inventors on an international patent application for "MultiSpecific Antibodies with Affinity for Human A33 Antigen and DOTA Metal Complex and Uses Thereof," filed on February 9, 2016. No other potential conflict of interest relevant to this article was reported. 


\section{ACKNOWLEDGMENTS}

We gratefully acknowledge Blesida Punzalan for her technical assistance. We are also grateful to Leah Bassity for her editorial assistance.

\section{REFERENCES}

1. Larson SM, Carrasquillo JA, Cheung NK, Press OW. Radioimmunotherapy of human tumours. Nat Rev Cancer. 2015;15:347-360.

2. Scott AM, Lee FT, Jones R, et al. A phase I trial of humanized monoclonal antibody A33 in patients with colorectal carcinoma: biodistribution, pharmacokinetics, and quantitative tumor uptake. Clin Cancer Res. 2005;11: 4810-4817.

3. Chong G, Lee FT, Hopkins W, et al. Phase I trial of ${ }^{131}$ I-huA33 in patients with advanced colorectal carcinoma. Clin Cancer Res. 2005;11:4818-4826.

4. Reardan DT, Meares CF, Goodwin DA, et al. Antibodies against metal chelates. Nature. 1985;316:265-268

5. Axworthy DB, Reno JM, Hylarides MD, et al. Cure of human carcinoma xenografts by a single dose of pretargeted yttrium- 90 with negligible toxicity. Proc Natl Acad Sci USA. 2000;97:1802-1807.
6. Orcutt KD, Slusarczyk AL, Cieslewicz M, et al. Engineering an antibody with picomolar affinity to DOTA chelates of multiple radionuclides for pretargeted radioimmunotherapy and imaging. Nucl Med Biol. 2011;38: 223-233.

7. Orcutt KD, Nasr KA, Whitehead DG, Frangioni JV, Wittrup KD. Biodistribution and clearance of small molecule hapten chelates for pretargeted radioimmunotherapy. Mol Imaging Biol. 2011;13:215-221.

8. Cheal SM, Xu H, Guo HF, et al. Theranostic pretargeted radioimmunotherapy of colorectal cancer xenografts in mice using picomolar affinity ${ }^{86} \mathrm{Y}-$ or ${ }^{177} \mathrm{Lu}-$ DOTA-Bn binding scFv C825/GPA33 IgG bispecific immunoconjugates. Eur J Nucl Med Mol Imaging. 2016;43:925-937.

9. Schlom J, Siler K, Milenic DE, et al. Monoclonal antibody-based therapy of a human tumor xenograft with a ${ }^{177}$ lutetium-labeled immunoconjugate. Cancer Res. 1991;51:2889-2896.

10. Wild D, Frischknecht M, Zhang H, et al. Alpha- versus beta-particle radiopeptide therapy in a human prostate cancer model $\left({ }^{213} \mathrm{Bi}\right.$-DOTA-PESIN and ${ }^{213} \mathrm{Bi}-$ AMBA versus ${ }^{177}$ Lu-DOTA-PESIN). Cancer Res. 2011;71:1009-1018.

11. Marks LB, Yorke ED, Jackson A, et al. Use of normal tissue complication probability models in the clinic. Int J Radiat Oncol Biol Phys. 2010;76(3, suppl): S10-S19.

12. Barendswaard EC, Humm JL, O'Donoghue JA, et al. Relative therapeutic efficacy of ${ }^{125} \mathrm{I}$ - and ${ }^{131} \mathrm{I}$-labeled monoclonal antibody A33 in a human colon cancer xenograft. J Nucl Med. 2001;42:1251-1256. 